\section{A PESCA DO CAMARÃO-DA-AMAZÔNIA, PERSPECTIVAS FUTURAS NO LITORAL PARAENSE}

\section{Bianca Bentes, Jussara M. Martinelli-Lemos, Camila Araújo e Victoria Isaac}

Os camarões Palaemonidae são conhecidos popularmente como camarões-de-água-doce (1). Os gêneros Palaemon, Palaemonetes e Macrobrachium são os mais representativos no Brasil, sendo este último o que mais se destaca por abranger 19 espécies (2), dentre as quais o camarão-da-Amazônia, Macrobrachium amazonicum, desperta interesse comercial principalmente nos estados do Pará, Amapá e Maranhão.

Embora já se tenha um pacote tecnológico desenvolvido especialmente para o cultivo de M. amazonicum, há uma carência muito grande de estudos voltados para a compreensão da dinâmica populacional e das relações ecológicas deste camarão em ambiente natural (3).

O crescente esforço das capturas de $M$. amazonicum na região Norte do Brasil já foi alertado por Lucena-Frédou e colegas (4), onde os autores chamam atenção para a falta de manejo de pesca para a espécie, bem como para o estabelecimento mínimo de tamanho para sua comercialização. Para haver um manejo dos recursos pesqueiros é fundamental que esse processo ocorra de forma colaborativa e integrando todos os atores e setores de interesse, isto é, tanto o governo, como o setor produtivo. Nesse contexto o presente artigo traz algumas abordagens a respeito da pesca do camarão-da-Amazônia, levando em consideração os aspectos biológicos, econômicos e sociais, a fim de discutir as questões sobre o futuro do estoque desse importante recurso pesqueiro.
PESCA E ESTUDOS SOBRE A ESPÉCIE O camarão-da-Amazônia é capturado de várias maneiras na zona costeira e águas interiores do estado do Pará. Entretanto, a utilização de armadilhas popularmente conhecidas como matapis parecem ser as mais utilizadas. Este petrecho é confeccionado com varetas finas de talas das palmeiras juba (Astrocaryum spp. e Atrix spp.) ou jupati (Raphia vinifer), amarradas com um traçado de cipó, formando uma armação cilíndrica que é fechada em cada extremidade em forma de funil (5). A isca utilizada para a pesca é o fruto da palmeira babaçu (Orbignya speciosa), vendida na forma de farinha nos mercados regionais (6).

É importante que haja um manejo adequado quanto à utilização dessa armadilha, uma vez que, em função da distância entre as talas, os matapis podem ser pouco seletivos dado que capturam camarões de diferentes tamanhos e faixas etárias, além do impacto da extração da palmeira nativa da floresta.

A reprodução de M. amazonicum é do tipo contínua ou periódica, com um aumento gradativo nos meses mais chuvosos. Os locais mais abrigados, como por exemplo as cabeceiras de canais-de-maré ou furos (comuns no estuário amazônico), são os locais preferencialmente procurados por fêmeas maduras para a desova e crescimento dos juvenis $(7 ; 8)$.

Estudos de crescimento e de avaliação do status de explotação na área mostraram que esses camarões crescem rapidamente, embora não atinjam grandes tamanhos. As taxas de mortalidade e explotação obtidas apontam para um estado de sobreexplotação do recurso, ou seja, em muito, já foi superada a taxa máxima de exploração sustentável (9).

PESCADORES E MANEJO PESQUEIRO A situação socioeconômica dos pescadores não é diferente dos demais sistemas pesqueiros do litoral do Pará (3). Bentes e colegas (10), ao entrevistarem 25 pescadores na Ilha de Mosqueiro em 2009, 


\section{Artigos $>$ Ensaios}

observaram a falta de níveis adequados de escolaridade; um manejo inexistente; dificuldade de obtenção e/ou administração dos financiamentos obtidos para a compra de petrechos e/ou embarcações; atuação deficiente das entidades de classe em representar a categoria; a existência de uma cadeia de comercialização difusa e pouco eficiente, uma vez que não valoriza os seus principais atores - os pescadores; e, por último, a falta de recolhimento de impostos oriundos da atividade de captura de M. amazonicum.

Ainda não existem políticas públicas voltadas ao ordenamento pesqueiro do camarão-da-Amazônia, entretanto, algumas iniciativas, com algum sucesso, têm sido realizadas. O camarão-da-Amazônia é, acima de tudo, mais um recurso tratado com descaso que tem mostrado sinais de sobreexplotação. A atividade de captura descontrolada, mesmo por pescadores artesanais, já está comprometendo os estoques pesqueiros (14).

Neste sentido, o que se observa são problemáticas de ordem social, ecológica e econômica que deveriam ser tratadas com maior cuidado. O problema social advém da introdução de mão de obra infantil na atividade, pois os filhos de pescadores, muitas vezes, diante da ausência de recursos, são obrigados a trabalhar na pesca cada vez mais cedo, seja na captura ou num primeiro "beneficiamento" - como é chamada a atividade de separação dos espécimes em "classes" (grandes, médios e pequenos) e a limpeza da produção. A questão ecológica se coloca dado o pouco conhecimento sobre a biologia dessa espécie e ainda pelo hábito do consumidor em comprar produtos com tamanho abaixo do estabelecido por lei, o que vem garantindo a sustentação desse mercado ilegal (venda de camarões jovens a menores preços). E a problemática econômica está no fato da atividade gerar rendimentos que são desconhecidos quanto à parte exportada para outros locais do Brasil, pois não são gerados impostos nem para o município, nem para o estado (3).

Essa situaçãoécomumentre a maioria dos recursos pesqueiros do Pará e gera, também, um outro problema econômico, pois, normalmente, esses recursos são exportados para outros locais (do Brasil e do mundo) como matéria-prima, sem nenhum tipo de beneficiamento que vise à agregação de valor ao produto como forma de converter rendimentos para todos os envolvidos na cadeia produtiva. Desta forma, o Pará permanece como mero exportador de matéria bruta e, além dos impostos não serem recolhidos no estado, o lucro jamais chega ao fornecedor primário - o pescador.
Trabalhos científicos contribuem para a geração de conhecimento sobre a espécie, porém é sabido que os recursos públicos para esse fim são de difícil acesso ou demandam tempo para a divulgação dos resultados. Adicionalmente, a coleta de informações de produção de camarão é deficiente, face aos inúmeros gargalos de sua cadeia de comercialização. Essa dificuldade se vale da grande quantidade de portos pesqueiros e dos volumes desembarcados. Esse fato, aliado à utilização de metodologias de extrapolação da produção cuja aplicabilidade não condiz aos sistemas pesqueiros paraenses, ainda culminam em dados pouco precisos da atividade pesqueira local. Desta forma, parece evidente a necessidade de um controle mais coerente da produção do camarão-da-Amazônia.

DIRETRIZES PARA UM MODELO BIOECONÔMICO Atualmente, vêm sendo utilizadas técnicas sofisticadas com excelente aplicabilidade no manejo pesqueiro. Essas ferramentas, conhecidas como modelos bioeconômicos ou análise de coortes, permitem delinear formas mais acuradas de manejo e delimitar possíveis cenários futuros, mediante a introdução de dados biológicos e/ou econômicos (3).

Para que um ideal de manejo seja atingido, a elaboração de um modelo bioeconômico precisa ser necessariamente validada. Esta validação, em se tratando do camarão-da-Amazônia, deve ser baseada nas consequências econômicas e biológicas da variação no esforço de pesca. Esses modelos são considerados instrumentos analíticos e vêm sendo utilizados no ordenamento de algumas espécies de peixes capturadas em países como o Chile e o Peru (12). Para o camarão-da-Amazônia, assim como para as demais espécies nas quais se pretende utilizar essa ferramenta, o modelo bioeconômico elaborado deve ser relativamente fácil de compreender e manejar e, ao mesmo tempo, ser bastante flexível, podendo adaptar-se facilmente ao volume de dados disponíveis. É importante, também, que todos os parâmetros possam mudar para que sejam mostrados os cenários/condições desse sistema pesqueiro, de modo que possam ser previstos cenários futuros das pescarias. Esse modelo - baseado em submodelos ecológicos, econômicos, tecnológicos e sociais - seria elaborado como mapa conceitual para o entendimento global do sistema pesqueiro. Para o camarão-da-Amazônia, um modelo bioeconômico deveria pelo menos compreender os aspectos mostrados na Figura I. 
Figura 1: Modelo conceitual de ferramenta bioeconômica de pescarias artesanais do camarão-da-Amazônia (Macrobrachium amazonicum) para a baía do Guajará, Pará. Fonte: Bianca Bentes

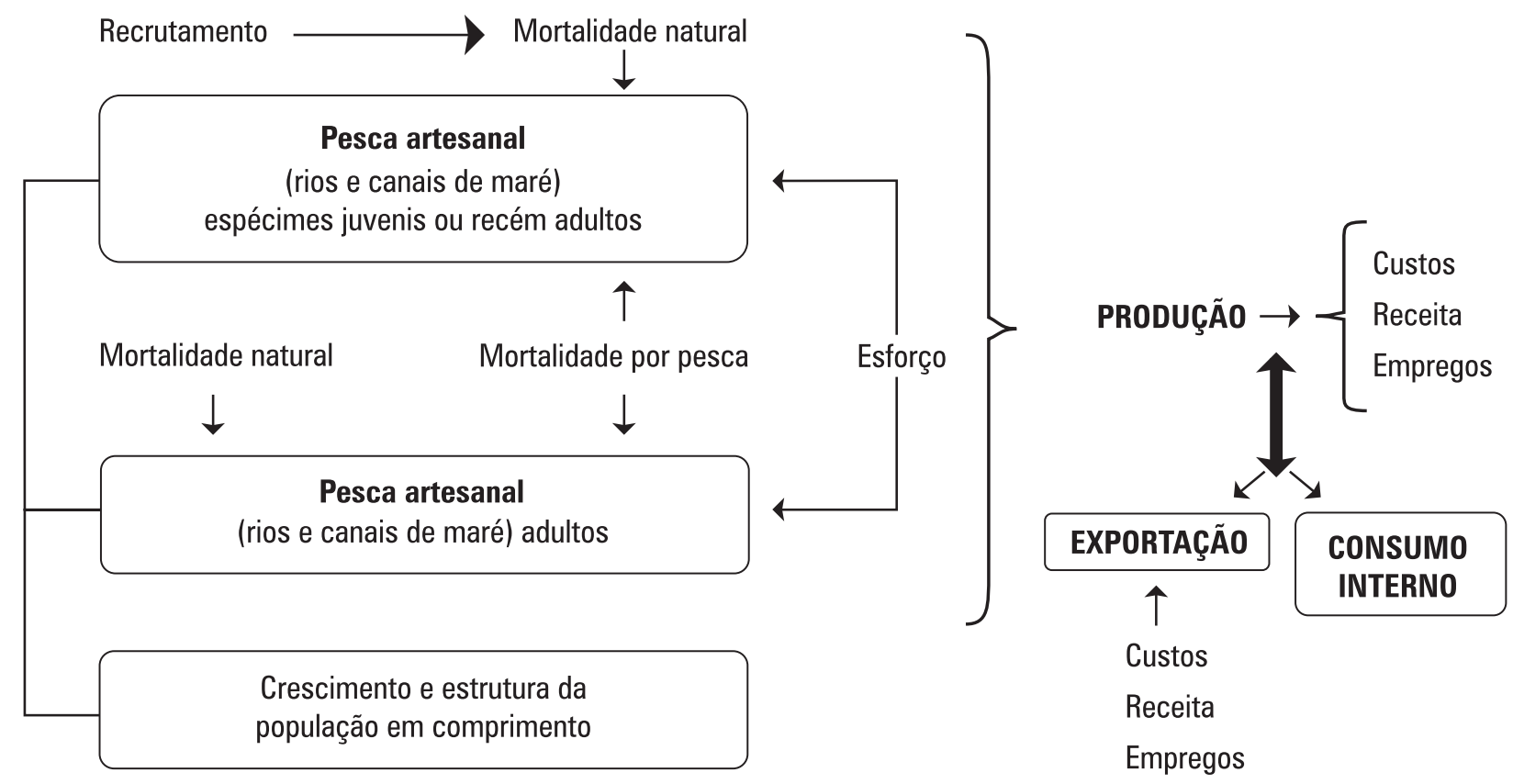

A avaliação dos efeitos econômicos, por exemplo, de um aumento ou diminuição da produção em um determinado setor, uma expansão ou contração da pescaria, poderia ser realizada mediante uma análise de insumo/produto (IP), que não se aplicaria somente à atividade de captura, mas a todas as outras etapas, uma vez que todas estariam conectadas. Por conseguinte, todos os demais efeitos poderiam ser previstos. O principal desfecho da análise seria, então, avaliar a eficácia de uma pescaria. Certamente, essas análises são muito pertinentes ao setor pesqueiro, onde os recursos são de uso comum e tendem a causar uma distribuição desigual (ou sub-ótima) dos fatores.

O modelo permitiria avaliar um nível ótimo de atuação do esforço para aumentar ao máximo os benefícios totais das capturas. Entretanto, para que isso aconteça é preciso, necessariamente, ter os dados claramente definidos. Outro importante papel para a otimização das pescarias e, consequentemente, de todo o sistema pesqueiro, é o da administração de todo o processo que, segundo Bossier e Wolff (16), deve se apoiar primeiramente a) na conservação, em longo prazo, dos recursos; b) na maximização da produção e da receita total das capturas ou da entrada de divisas; c) na maximização do lucro; d) na diminuição dos custos de produção; e) na melhoria da condição econômica e social da mão de obra, para uma melhor oportunidade de emprego e redistribuição dos benefícios.

Certamente, esses seriam os princípios básicos para uma administração pesqueira ideal, entretanto, é sabido que, no caso específico de camarões, como ressaltam Isaac e colegas (17), existem peculiaridades que dificultam esse processo, em particular o fato de que os camarões apresentam elevados valores na comercialização - o que já pode ser observado também para M. amazonicum nas feiras de mercados populares de Belém (PA) - fato que aumenta extraordinariamente o incentivo para aumento de esforço de pesca. Assim, a falta de uma adequada administração da atividade, desde seu princípio, pode vir a possibilitar esse incremento demasiadamente elevado do esforço de pesca, conduzindo à rentabilidade nula e até a uma redução dos valores de capturas totais. Fortalece-se a necessidade da administração dessas pescarias, o que pode ser viabilizado utilizando-se os modelos bioeconômicos supracitados, que convergem para a visualização desses possíveis cenários futuros mediante a introdução de dados biológicos ou da geração de divisas no modelo, por exemplo.

A baía do Guajará é um ambiente de fácil acesso à fiscalização e monitoramento da atividade, tendo vista a proximidade desta com a capital e a temporalidade dos desembarques 
que seguem o ritmo das marés. Sendo assim, a normalização das capturas de camarão-da-Amazônia certamente teria sucesso, porém, a limitação do acesso para a pescaria como, por exemplo, um licenciamento dos pescadores artesanais que pudessem atuar na área, tenderia a provocar a transferência do excesso do esforço para as pescarias ou estoques de áreas vizinhas e, provavelmente, teria que ser limitado também o acesso em todas as pescarias próximas, com o objetivo de controlar essa transferência. Esta problemática já havia sido discutida por Garcia e Le Reste (18). Além disso, para essa baía em particular, a falta de um cadastro de pescadores, que viabilizasse um monitoramento das pescarias, é um impedimento significativo para se implantar as tentativas de controle direto do esforço.

Uma alternativa favorável à diminuição da pressão dos estoques naturais seria o incentivo ao cultivo da espécie, o que já vem sendo observado entre os próprios pescadores que fazem um certo "controle" do pescado, mantendo os menores espécimes em viveiros, sendo um indicativo de que, se fossem orientados corretamente, poderiam ajudar no manejo do recurso.

Uma necessidade imediata é o financiamento para a realização de pesquisas que contemplem estudos básicos da biologia dessa espécie em outras áreas do estado ou da Amazônia como um todo, como forma de integrar conhecimentos e avaliar a repercussão de investimentos no manejo in situ e ex situ. Como um primeiro passo, a Secretaria de Estado de Pesca e Aquicultura (Sepaq/PA) aprovou, em 2009, uma rede de pesquisa com algumas instituições de todo o Brasil, com temáticas voltadas à pesquisa em ambiente natural e no cultivo de M. amazonicum, denotando novamente a importância do recurso. Os trabalhos consistem no desenvolvimento de pesquisas para otimizar a produção da larvicultura, pois essa é a fase mais complexa do cultivo. As expectativas, até então, são positivas uma vez que os avanços alcançados objetivam viabilizar a interação entre a pesquisa e o setor produtivo.

Bianca Bentes é professora adjunta do Instituto de Estudos Costeiros da Universidade Federal do Pará (UFPA).Email: bianca_bl@yahoo.com.br

Jussara Martinelli-Lemos é professora associada do Instituto de Ciências Biológicas e coordenadora do Programa de Pós-Graduação em Ecologia Aquática e Pesca da UFPA.

Camila Araújo é mestranda do curso de pós-graduação em biologia ambiental do Instituto de Estudos Costeiros da UFPA e membro do Grupo de Pesquisas em Ecologia de Crustáceos da Amazônia.
Victoria Isaac é professora associada do Instituto de Ciências Biológicas e coordenadora do Laboratório de Biologia Pesqueira e Manejo de Recursos Aquáticos da UFPA.

\section{REFERÊNCIAS}

1. Holthuis, L.B. "A general revision of the Palaemonidae (Crustacea Decapoda Natantia) of the Americas." Allan Hancock Foundation Publications. Occasional Paper. The subfamily Palaemoninae. University of Southern California, p 1-395. 1952.

2. Bauer, R.T. Remarkable shrimps: adaptations and natural history of the Carideans. University of Oklahoma Press, Norman, 316p. 2004.

3. Bentes, B. S. "Ecologia, pesca e dinâmica populacional do camarão-da-Amazônia - Macrobrachium amazonicum (Heller, 1862) (Decapoda: Palaemonidae) - capturado na região das ilhas de Belém - Pará - Brasil". Tese de doutorado. Universidade Federal do Pará, Belém. 256p. 2011.

4. Lucena-Frédou, F.; Rosa, J. S.; Silva, M. C. N \& Azevedo E.F. "Population dynamics of the River Prawn, Macrobrachium amazonicum (Heller, 1862) (Decapoda, Palaemonidae) on Combu island (Amazon estuary)". Crustaceana, pp. 277-290. 2010.

5. Odinetz Collart, O. \& Magalhães, C. "Ecological constraints and life history strategies of Palaemonidae Prawns in Amazonia". Verhandlungen Internationale Vereinigung fur theoretische und angewandte limnologie, 25: 2460-2467. 1994.

6. Azevedo, E. F. "Biologia reprodutiva do camarão regional Macrobrachium amazonicum (Heller, 1862) (Decapoda; Palaemonidae), na ilha do Combu (Belém, PA)". Trabalho de conclusão de curso. Universidade Federal do Pará. 28p. 2004.

7. Bentes, B.; Martinelli-Lemos, J. M.; Cardoso, C. N. A.; Nascimento, M. S.; Isaac, V. J. N. Reproductive biology of Macrobrachium amazonicum (Heller, 1862) in an estuary in the Brazilian Amazonian. 2016. (no prelo).

8. Bentes, B. S.; Martinelli, J. M.; Souza, L. S.; Cavalcante, D.V.; Almeida, M. C.; Isaac, V. "Spatial distribution of the amazon river prawn Macrobrachium amazonicum (Heller, 1862) (Decapoda, Caridea, Palaemonidae) in two perennial creeks of an estuary on the Northern coast of Brazil (Guajará Bay, Belém, Pará)". Brazilian Journal of Biology, 71(4): 925-935. 2011.

9. Peixoto, S. N. B. "Caracterização molecular e biologia reprodutiva de Macrobrachium amazonicum (Crustacea, Decapoda, Palaemonidae)". Dissertação de Mestrado. Universidade Federal do Pará, 71p. 2002.

10. Bentes, B.; Cañete, V. R.; Pereira, L. J. G.; Martinelli-Lemos, J. M.; Isaac, V. "Descrição socioeconômica da pesca do camarão Macrobrachium amazonicum (Heller, 1862) (Decapoda: Palaemonidae) em um estuário da costa Norte do Brasil: o caso da ilha de Mosqueiro (PA)". Boletim do Laboratório de Hidrobiologia, 25: 21-30. 2012.

11. Bentes, B. S.; Isaac, V. J.; Martinelli-Lemos, J. M. Dinâmica populacional do camarão-da-Amazônia-M. amazonicum (Heller, 1862) (Decapoda: Palaemonidae) - no estuário Guajará - Belém - Brasil. 2016. (no prelo).

12. Bossier, S.; Wolff, M.; Soetaert, K. "Causes of performace differences between scallop culture in Peru and Chile: a bio-economical modeling approach.” In: Mees, J. et al. (Ed.) (2015). Book of abstracts - VLIZ young scientists' day, Brugge. VLIZ Special Publication, 26p. 2015.

13. Isaac, V. J.; Dias Neto, J.; Damasceno, F. G. "Camarão rosa da costa Norte. Biologia, dinâmica e administração pesqueira”. Coleção Meio Ambiente. Ibama. Série Estudos Pesca. 187p. 1992.

14. Garcia, S.; Le Reste, L. "Life cycles, dynamics, explotation and management of coastal penaeid shrimp stocks”. FAO Fisheries Technology Papers, pp. 203-215. 1981. 